\title{
Kommunale Selbstverwaltung in der Zivilgesellschaft
}

\section{Plädoyer für die bürgerschaftliche Revitalisierung einer modernen Institution}

Holger Backhaus-Maul

Holger Backhaus-Maul leitet das Fachgebiet Recht, Verwaltung und Organisation an der Fakultät für Erziehungswissenschaften der Martin-Luther-Universität HalleWittenberg. Internet http://www.philfak3.unihalle.de/paedagogik/rvo
Die Entwicklung einer in der Zivilgesellschaft verankerten kommunalen Daseinsvorsorge ist voraussetzungsreich, denn bürgerschaftliches Engagement braucht institutionelle Entwicklung und Förderung. Dazu müssen Kommunen einerseits die erforderlichen Ressourcen erhalten und andererseits die Stärkung der Position des Bürgers als Auftraggeber, Mitgestalter und Kunde auch wollen.

Die Institution der kommunalen Selbstverwaltung ist eine zivilgesellschaftliche Errungenschaft, deren sozialkulturelles Potenzial bisweilen leichtfertig verkannt wird. So wird über kommunale Selbstverwaltung zumeist erst dann diskutiert, wenn - wieder einmal - staatliche Vorgaben und Aufgabenüberwälzungen im Gleichklang mit unzulänglichen föderalen Finanzzuweisungen die in Artikel 28 des Grundgesetzes verfassungsrechtlich verbriefte Gestaltungs- und Innovationsfähigkeit deutscher Städte und Landkreise in allen Angelegenheiten der öffentlichen Daseinsvorsorge infrage stellen (grundlegend Wollmann 1990; Pitschas 2003, Backhaus-Maul 2009).

Angesichts latenter fiskalischer und politischer Restriktionen richten sich die öffentliche Aufmerksamkeit sowie der Protest und die "Mitleidsbekundungen " in erster Linie an Kommunalpolitik und Kommunalverwaltung. Dabei geraten aber der originäre Anlass und die sozialkulturellen Grundlagen kommunaler Selbstverwaltung leicht aus dem Blick.

Als politische Errungenschaft eröffnet kommunale Selbstverwaltung nämlich Bürgern einerseits weitreichende Möglichkeiten zur Entscheidung und Gestaltung in den sie betreffenden Angelegenheiten der örtlichen Daseinsvorsorge. Andererseits bildet die kommunale Selbstverwaltung den institutionellen Rahmen dafür, dass Bürger freiwillig eigene sachliche, zeitliche und monetäre Ressourcen in die Entwicklung des Ge- meinwesens einbringen können. Aber die in Demokratien überaus fruchtbare Synthese aus Politik und Engagement, aus Mitentscheiden und Mittun war in vergangenen Jahren angesichts staatlich vorgegebener Bedingungen vielerorts einer tiefgreifenden Sinnentleerung ausgesetzt.

Das aktuelle Dilemma und die Restriktionen kommunaler Selbstverwaltung sind das Ergebnis vorangegangener staatlicher - Entscheidungen, die die Rahmenbedingungen kommunaler Selbstverwaltung seit einigen Jahren und Jahrzehnten grundlegend verändert haben.

Zeithistorisch ist zunächst in Rechnung zu stellen, dass Föderalismus und kommunale Selbstverwaltung nach dem deutschen Faschismus institutionelle Antworten auf Zentralisierung und Monopolisierung von Macht und deren Missbrauch sowie Ansätze zur demokratischen ReEducation Deutschlands waren. Heutzutage erscheint in einer globalisierten Welt ein derart hierarchisch fein abgestuftes Mehrebenensystem, das Macht und Geld horizontal und vertikal ausdifferenziert zwischen Bund, Ländern und Kommunen verteilt, als parzelliert und zu behäbig. Das Bild von einer organisierten und zugleich fragmentierten Verantwortungslosigkeit drängt sich auf. Zugleich geraten Föderalismus und kommunale Selbstverwaltung seit Jahrzehnten zusehends in Auseinandersetzungen mit politisch engagierten, fachlich kompetenten und sich ihrer Souveränität bewussten Bürgern.

Angesichts von Globalisierung und politischem Bürgerengagement sind Kommunen spätestens seit den 1970er Jahren zusehends in eine Handlungsklemme geraten: Während politische Entscheidungen beim Bund zentralisiert werden, wird Kommunen die konkrete Aufgabenerledigung zugewiesen. Erinnerungen an die Vorstellungswelt des Subsidiaritätsprinzips, dem ordnungspolitischen Vorrang der jeweils kleinsten leistungsfähigen Einheit bei der Bewältigung gesellschaftlicher 
Aufgaben, können angesichts dieser Entwicklungen heutzutage fast schon Befremden auslösen. Und das in einigen Landesverfassungen formulierte und überaus sinnvolle Konnexitätsprinzip, das die staatliche Aufgabendelegation mit der Verpflichtung zu einer hinreichenden staatlichen Finanzierung verknüpft, bringt staatlicherseits eher ein schlechtes Gewissen zum Ausdruck, als dass es bisher den Geist kommunaler Selbstverwaltung merklich wiederbeleben konnte.

Diese sukzessive Entwertung von Kommunen ist das Ergebnis staatlicher Entscheidungen über die institutionelle Gestaltung der kommunalen Selbstverwaltung und die Art und Weise der kommunalen Daseinsvorsorge. Die Institution der kommunalen Selbstverwaltung als Kernbestandteil der repräsentativen Demokratie und als modernes bürokratisches und professionelles Organisationsprinzip wurde nach dem Zweiten Weltkrieg in den alten und 1990 in den neuen Bundesländern eingeführt.

Im Zuge der Diskussion über neue betriebswirtschaftlich inspirierte - Steuerungsmodelle in der öffentlichen Verwaltung wurden vor allem Kommunalverwaltungen anhand von Kriterien und Verfahren der Effizienzmessung flächendeckend an staatlichen Vorstellungen von Wirtschaftlichkeit ausgerichtet (vgl. Jann 2011). Zeitgleich wurde zumindest vereinzelt bereits auf die einseitige wirtschaftliche Ausrichtung und das Demokratiedefizit neuer Steuerungsmodelle hingewiesen.

Die Idee der Bürgerkommune bringt diese Vorstellung von einer partizipativen Öffnung neuer Steuerungsmodelle zum Ausdruck. Die bisher vorliegenden Bilanzierungen der Implementation neuer Steuerungsmodelle und der Idee der Bürgerkommune kommen insgesamt zu bemerkenswerten Befunden: Das institutionelle Beharrungsvermögen der kommunalen Selbstverwaltung hat eine höchst selektive und unterschiedliche Rezeption der genannten Modelle und Ideen zur Folge (vgl. Bogumil/Grohs/Kuhlmann 2007).

Kommunalverwaltung und Kommunalpolitik, aber auch lokale Gemeinwesen gründen zumeist im manifesten Willen zur Machterhaltung und einer latenten Zurückhaltung gegenüber Veränderungen und Umbrüchen. Auf kommunaler Ebene erfolgen Veränderungen auf inkrementeller Art und Weise, also unter Rückgriff auf Gewohntes und Bewährtes sowie langsam und bedächtig. Inkrementelles Lernen bedeutet anderseits aber auch, dass entsprechende institutionelle Veränderungen stabil und von Dauer sind.

Vor diesem Hintergrund sind die Bilanzierungen der neuen Steuerungsmodelle auf kommunaler Ebene von Jörg Bogumil und Lars Holtkamp zu bewerten, die eine Stärkung der Position des Bürgers als Kunde öffentlicher Dienstleistungen sowie als Auftraggeber und Mitgestalter kommunaler Selbstverwaltung konstatie- zum interkommunalen Standortwettbewerb leisten.

Die Potenziale bürgerschaftlichen Engagements auf kommunaler Ebene bleiben aber angesichts der Singularität, Fragmentierung und Fragilität der Projekte und Ansätze bürgerschaftlichen Engagements weit hinter ihren faktischen Möglichkeiten zurück. So sind Engagementprojekte in der Regel nicht Ausdruck einer vitalen Zivilgesellschaft, sondern zumeist das schlichte Ergebnis nicht aufeinander abgestimmter oder absichtsvoll miteinander konkurrierender öffentlicher Förderprogramme. In der zeitlichen Ab-

\section{»Engagementprojekte sind oft nicht Ausdruck einer vitalen Zivilgesellschaft, sondern das Ergebnis öffentlicher Förderprogramme«}

ren (vgl. Bogumil/Holtkamp 2010). Die Stärkung der Position des Bürgers kommt - so die langjährigen empirisch gehaltvollen Beobachtungen von Gisela Jakob nicht zuletzt in diversen Versuchen, Ansätzen und Projekten bürgerschaftlichen Engagements auf lokaler Ebene zum Ausdruck (vgl. Jakob 2010). Die Spannbreite der erfolgreichen und teilweise auch gescheiterten Versuche bürgerschaftlichen Engagements reicht dabei von Selbsthilfekontaktstellen und Seniorenbüros über Freiwilligenagenturen und Bürgerstiftungen bis hin Bündnissen für Familien und Mehrgenerationenhäusern.

Als wesentliche Gründe für die aktuelle Vielfalt und Vielzahl von Aktivitäten bürgerschaftlichen Engagements auf lokaler Ebene sind einerseits schlichte Notwendigkeiten und andererseits die im Zuge von steigender Bildung und wachsendem Wohlstand zunehmenden Kompetenzen und Ressourcen von Bürgern anzuführen. Neben wirtschaftlichen und politischen Faktoren sind es gerade diese sozialkulturellen Grundlagen kommunaler Selbstverwaltung, vor allem in den Ausprägungen des bürgerschaftlichen Engagements, die einen wesentlichen Beitrag zur lokalen Wohlfahrtsproduktion und folge dieser Förderprogramme kommt es oftmals zu grundlegenden Schwerpunktveränderungen oder zu neuen programmatischen Akzentsetzungen, die letztlich nicht eine lokale Engagement-Infrastruktur entstehen lassen, sondern sachlich, zeitlich und sozial begrenzte Einzelmaßnahmen, Projekte und Initiativen ins Leben rufen, um sie nach einer kurzen Förderphase in den "unendlichen Weiten des engagementpolitischen Raumes" sich selbst zu überlassen. Derart geförderte Engagementprojekte können sich so geradezu glücklich schätzen, wenn sie mehr oder minder zufällig in einer Kommune angesiedelt sind, die gesellschaftspolitisch bestrebt ist, ihre eigenen sozialkulturellen Grundlagen im Rahmen kommunaler Selbstverwaltung zu entwickeln. Unsere soeben im Auftrag des Bundesministeriums für Familie, Senioren, Frauen und Jugend abgeschlossene empirische Untersuchung über die Entwicklung von Freiwilligenagenturen und Freiwilligenzentren in Deutschland veranschaulicht dieses Dilemmata materialreich.

Aber die Entwicklung der sozialkulturellen Grundlage einer Kommune ist voraussetzungsreich. Die immer wieder von Engagementexperten vorgetragene Ko- 
operationserwartung ist günstigstenfalls gut gemeint, bleibt aber gesellschaftspolitisch folgenlos, wenn nicht einmal basale institutionelle Rahmenbedingungen bürgerschaftlichen Engagements gegeben sind und es an organisierten Akteuren und einem erklärten politischen Wille fehlt.

Bürgerschaftliches Engagement - verstanden als Kernbestandteil der sozialkulturellen Grundlagen kommunaler Selbstverwaltung - erschließt sich erst in

\section{Literatur}

Backhaus-Maul, Holger 2009: Sozialpolitische Entwicklungslinien in Deutschland, in: Ulli Arnold, Bernd Maelicke (Hg.), Lehrbuch der Sozialwirtschaft, Baden-Baden, S. 96-116.

Bogumil, Jörg/Grohs, Stephan/Kuhlmann, Sabine 2007: Zehn Jahre Neues Steuerungsmodell - eine Bilanz kommunaler Verwaltungsmodernisierung, Berlin.

Bogumil, Jörg/Holtkamp, Lars 2010: Die kommunale Ebene, in: Thomas Olk, Ansgar Klein, Birger Hartnuß (Hg.), Engagementpolitik. Die Entwikklung der Zivilgesellschaft als politische Aufgabe, Wiesbaden, S. 382-403.

Jakob, Gisela 2010: Infrastrukturen und Anlaufstellen zur Engagementförderung in den Kommunen, in: Thomas Olk, Ansgar Klein, Birger Hartnuß (Hg.), Engagementpolitik. Die Entwikklung der Zivilgesellschaft als politische Aufgabe. Wiesbaden, S. 233-259.

Jann, Werner 2011: Neues Steuerungsmodell, in: Bernhard Blanke, Frank Nullmeier, Christoph Reichard, Göttrik Wewer (Hg.), Handbuch zur Verwaltungsreform, Wiesbaden, S. 98108.

Pitschas, Rainer 2003: Kommunale Sozialpolitik, in: Bernd von Maydell, Franz Ruland (Hg.), Sozialrechtshandbuch, Darmstadt, S. 1295-1335.

Wollmann, Hellmut 1990, Politik- und Verwaltungsinnovationen in den Kommunen? Eine Bilanz kommunaler Sozial- und Umweltschutzpolitik, in: Thomas Ellwein, Joachim Jens Hesse, Renate Mayntz, Fritz W. Scharpf (Hg.), Jahrbuch zur Staats- und Verwaltungswissenschaft, Baden-Baden, S.69-112. einer institutionentheoretischen Perspektive und erfordert eine entsprechende institutionelle Entwicklung und Förderung. Im Sinne des ordnungspolitischen Subsidiaritätsprinzips wären Kommunen zunächst mit der notwendigen Entscheidungsautonomie in den sie betreffenden Angelegenheiten sowie im Sinne des Konnexitätsprinzips mit den erforderlichen monetären Ressourcen auszustatten. Nicht zuletzt aber ist auf Seiten von Kommunen der erklärte politische Wille zur Revitalisierung der kommunalen Selbstverwaltung, insbesondere zur Stärkung der Position des Bürgers als Auftraggeber, Mitgestalter und Kunde unabdingbar.

Erst unter diesen institutionellen Grundbedingungen und gesellschaftspolitischen Prämissen kann sich eine lokale Infrastruktur bürgerschaftlichen Engagements entwickeln und entfalten, die diesem anspruchsvollen Begriff gerecht werden kann.

\section{Altenpflege am Scheideweg!}

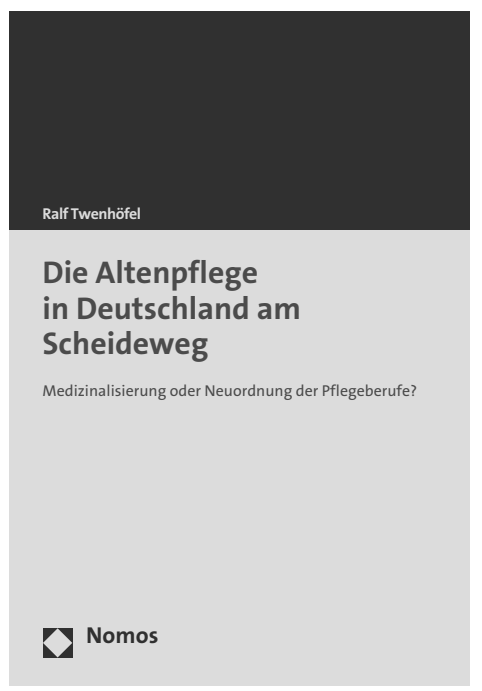

Die Untersuchung plädiert für eine Professionalisierung des Altenpflegeberufs. Sie wendet sich gegen einseitige, politisch derzeit mit Nachdruck unterstützte Bestrebungen einer „Medizinalisierung“, die Professionalität in erster Linie an der medizinisch-pflegerischen Qualifikation von Pflegekräften bemisst. Das Werk zeigt die Folgen einer derart restringierten Konstruktion in der Pflegepraxis auf und erörtert in einer systemtheoretischen Perspektive Erfordernisse und Möglichkeiten einer „Nursing“ und „Caring“ umfassenden Professionalisierung des Berufs. Nur eine solcherart „ganzheitliche“ professionelle Pflege würde, so die These, ihren Beitrag zur Lebensqualität von Pflegebedürftigen im Alter leisten können. Nur dann auch ließe sich im Einzelfall eine Entwicklung korrigieren, die die Aussicht bietet, dass die ,alternde“ Gesellschaft der Bundesrepublik eine Gesellschaft von Patienten wird.

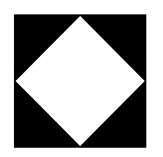

\section{Nomos}

Bitte bestellen Sie im Buchhandel oder

versandkostenfrei unter $\boldsymbol{w w w}$ w.nomos-shop.de 